Original Article

\title{
Effects of craniocervical flexion exercise on upper-limb postural stability during a goal-directed pointing task
}

\author{
Min-Sik Yong, PhD ${ }^{1)}$, Hae-Yong Lee, MS ${ }^{2)}$, Young-Uk Ryu, PhD ${ }^{3)}$, Mi-Young Lee, PhD ${ }^{4}$ * $^{*}$ \\ 1) Department of Physical Therapy, Youngsan University, Republic of Korea \\ 2) Department of Rehabilitation Science, Graduate School, Daegu University, Republic of Korea \\ 3) Department of Physical Therapy, College of Health Sciences, Catholic University of Daegu, \\ Republic of Korea \\ 4) Department of Physical Therapy, College of Health and Therapy, Daegu Haany University: 1 \\ Haanydaero Gyeongsan-si, Gyeongsangbuk-do, Republic of Korea
}

\begin{abstract}
Purpose] This study investigated the effects of craniocervical flexion exercise on upper-limb postural stability by measuring upper-limb postural tremor during a goal-directed pointing task. [Subjects and Methods] Twenty-four subjects were randomly assigned to the exercise or control group. The exercise group performed craniocervical flexion exercise four days per week for five weeks. Upper-limb postural tremor was measured by using a three-dimensional electromagnetic motion tracking system (trakSTAR ${ }^{\mathrm{TM}}$, Ascension Technology Corporation, Burlington, VT, USA) during a goal-directed pointing task. [Results] In the exercise group, the range and velocity of the trajectories of the shoulder, wrist, and finger in the lateral direction improved significantly. However, no significant changes were observed in the control group. [Conclusion] Craniocervical flexion exercise reduces the range and velocity of upper-limb postural tremor, thereby increasing postural stability.

Key words: Craniocervical flexion exercise, Postural stability, Proprioception
\end{abstract}

(This article was submitted Feb. 19, 2015, and was accepted Mar. 14, 2015)

\section{INTRODUCTION}

Postural control is defined as the control of the body's position to perform activities of daily living. Postural control for stability is accomplished by interactions between the musculoskeletal and nervous systems ${ }^{1)}$. As the cervical region contains a dense concentration of proprioceptive organs such as muscle spindles, it plays an importance role in providing afferent proprioceptive information for postural control $^{2-4)}$. However, this function of the cervical region is perturbed by neck pain, fatigue, abnormal proprioception, and poor movement patterns due to muscular imbalance, which can be observed in people exhibiting impaired postural control ${ }^{2,5)}$. Such perturbations consequently influence postural stability ${ }^{6}$.

Craniocervical flexion is generally used to test the activation of deep cervical flexor muscles, which play an important role in controlling cervical spine stability ${ }^{3}$. Coordination between the superficial and deep cervical flexor muscles can also be observed when subjects perform craniocervical

*Corresponding author. Mi-Young Lee (E-mail: mykawai@ hanmail.net)

(C2015 The Society of Physical Therapy Science. Published by IPEC Inc. This is an open-access article distributed under the terms of the Creative Commons Attribution Non-Commercial No Derivatives (by-ncnd) License $<$ http://creativecommons.org/licenses/by-nc-nd/3.0/>. flexion. In addition, craniocervical flexion can be used as an exercise; it not only aids pain relief, but also improves coordination between the superficial and deep cervical flexors ${ }^{7}$. Furthermore, Armstrong et al. ${ }^{2)}$ assert craniocervical flexion exercise affects the proprioceptive functions of the head and neck.

Previous studies involving craniocervical flexion exercise have been conducted to demonstrate the effects of exercise on patients with neck pain. However, few studies have investigated postural stability. Therefore, the present study investigated whether craniocervical flexion exercise affects upper-limb postural stability by measuring upper-limb postural tremor during a goal-directed pointing task.

\section{SUBJECTS AND METHODS}

A total of 24 healthy right-handed subjects with no history of neurological, biomechanical injury, or pain in the cervical region or upper limbs were recruited. All subjects were randomly assigned to the exercise group ( $n=10$, mean age, $23.6 \pm 1.4)$ or control group ( $n=14$, mean age, $23.4 \pm 1.4)$. The demographic data of the subjects are shown in Table 1 . The study protocol was approved by the Public Institutional Bioethics Committee designated by the MOHW. All subjects understood the purpose of this study and provided written informed consent prior to participation.

The subjects in the exercise group performed craniocervical flexion exercise four days per week for five weeks. The 
2006 J. Phys. Ther. Sci. Vol. 27, No. 6, 2015

Table 1. Demographic characteristics of subjects

\begin{tabular}{lcc}
\hline & Exercise group & Control group \\
\hline Gender (M/F) & $6 / 4$ & $5 / 9$ \\
Age (years) & $23.6 \pm 1.4$ & $23.4 \pm 1.4$ \\
Height $(\mathrm{cm})$ & $168.2 \pm 5.4$ & $166.30 \pm 7.3$ \\
Weight $(\mathrm{kg})$ & $60.9 \pm 9.1$ & $59.30 \pm 12.1$ \\
\hline \multicolumn{2}{c}{ Data are expressed as mean \pm SD. }
\end{tabular}

Data are expressed as mean $\pm \mathrm{SD}$.

craniocervical flexion exercise consisted of activation of the deep cervical flexor muscles and progressive upper limb exercise. The subjects assumed a crook-lying position with the neck in a neutral position. A pressure biofeedback unit (Stabilizer Pressure Biofeedback Unit, Chattanooga Group Inc., Hixson, TN, USA) was used for craniocervical flexion exercise to provide visual feedback to patients while training for deep muscle activation. The folded stabilizer pressure biofeedback unit was placed under the upper cervical spine and inflated to $20 \mathrm{mmHg}$. Then, the subjects performed a slow controlled nodding motion of the head on the upper cervical spine. The subjects were instructed to nod, increase the pressure on the cuff up to $22 \mathrm{mmHg}$, and maintain the pressure for 10 seconds. In addition, shoulder flexion, abduction, and horizontal adduction/abduction movements were performed during craniocervical flexion, progressing from a bilateral to reciprocal exercise. The pressure was set from $22-30 \mathrm{mmHg}$, which was subsequently increased by 2 $\mathrm{mmHg}$ above the baseline pressure to increase the exercise intensity. In the final weeks, the subjects performed craniocervical flexion during a shoulder exercise with light dumbbells; three sets of $12-15$ repetitions were performed. The test-retest reliability of the craniocervical flexion test has an intraclass correlation coefficient of 0.81 for the activation score and 0.93 for performance ${ }^{8,9)}$. Meanwhile, the controls performed daily activities as usual with no specific exercises.

To measure the change of upper-limb postural tremor induced by craniocervical flexion exercise, a goal-directed pointing task was performed before and after exercise. The subjects were examined in an upright standing posture $4 \mathrm{~m}$ from a target on a wall. The target consisted of six circles; the center was marked by a "+" sign. The subjects held a laser pointer between the pad of the thumb and mediolateral surface of the index finger. They stretched out their upper limb toward the target and pressed the button on the laser pointer. The subjects were instructed to hit and keep the laser emission on the center of the target for 30 seconds two times.

A three-dimensional electromagnetic motion tracking system was used for data acquisition (trakSTAR ${ }^{\mathrm{TM}}$, Ascension Technology Corporation, Burlington, VT, USA). Three markers were attached to well-known anatomical landmarks on the shoulder, wrist, and finger: the lateral board of shoulder acromion, styloid process of the ulna, and head of the second metacarpal bone, respectively. All evaluations were performed by the same experimenter in order to achieve intrasubject reproducibility. The positional coordinates of active markers are expressed in the lateral (i.e., y-axis) and vertical (i.e., z-axis) directions. Data were acquired at a sampling frequency of $100 \mathrm{~Hz}$.

MATLAB (The Mathworks, Natick, MA, USA) was used for data processing and analysis. The trajectories along the lateral and vertical directions were obtained and quantified for landmarks placed on the shoulder, wrist, and finger ${ }^{10)}$. The trajectory range and mean velocity of each marker were calculated using following formulae:

Trajectory range $=\mid \max ($ trajectory $)-\min ($ trajectory $) \mid$

Mean velocity $=\frac{\sum_{n=1}^{N-1}|\operatorname{traj}[n+1]-\operatorname{traj}[n]|}{T}$

Statistical analysis was performed using SPSS version 14.0 for Windows (SPSS Inc., Chicago, IL, USA). All normally distributed data were analyzed by a parametric test; the $\chi^{2}$ test or independent t-test was used to compare demographic data. An independent t-test was used to evaluate differences in the range and velocity of trajectories between the exercise and control groups. A paired t-test was used to determine differences before and after treatment in each group. The level of significance was set at $\mathrm{p}<0.05$.

\section{RESULTS}

The demographic characteristics did not differ significantly between the exercise and control groups ( $p>0.05)$. In the exercise group, the lateral direction of the range and velocity of the trajectories of the shoulder, wrist, and finger increased significantly $(p<0.05)$. However, there was no difference in the vertical direction of the range or velocity $(p>0.05)$. In the control group, there were no significant changes in any variable $(\mathrm{p}>0.05)$. In addition, intergroup comparison showed craniocervical flexion exercise significantly enhanced the lateral direction of the range and velocity of trajectory of the shoulder $(\mathrm{p}<0.05)$. The results are summarized in Tables 2 and 3.

\section{DISCUSSION}

The present study investigated the range and velocity of upper-limb postural tremor during a goal-directed pointing task to determine if craniocervical flexion exercise affects upper-limb postural stability. The results show the exercise group had a significantly lower range and velocity of upperlimb postural tremor than the control group.

Healthy people exhibit physiological tremor, which is involuntary and rhythmic limb motions. As it decreases the ability to perform fine movements, exertion is necessary to manage it. Controlling tremor involves maintaining optimal postural stability. In this regard, it is critical to determine how people can control their own tremor not only for improving the performance of tasks requiring fine movement, but also to contribute to postural stability ${ }^{10,11)}$.

The deep cervical flexor muscles play an important role in stabilizing the head-on-neck posture. Disruption of the balance between the posterior and anterior muscles in the neck can impede the maintenance of proper posture ${ }^{12)}$. Prolonged or exaggerated imbalance may contribute to muscle weakness as well as reduced muscle spindle sensitivity, resulting in reduced proprioception ${ }^{5,13-14)}$. Furthermore, Sanchez et al. ${ }^{6}$ report that such changes are associated with decreased postural stability. 
Table 2. Effects of craniocervical flexion exercise on the ranges of trajectories

\begin{tabular}{lccccc}
\hline & & \multicolumn{2}{c}{ Exercise group $(\mathrm{cm})$} & \multicolumn{2}{c}{ Control group $(\mathrm{cm})$} \\
\hline Lateral direction & Shoulder & $1.13 \pm 0.30$ & $0.62 \pm 0.29^{* \dagger}$ & $1.28 \pm 0.69$ & $1.15 \pm 0.59$ \\
(y-axis) & Wrist & $1.22 \pm 0.37$ & $0.80 \pm 0.36^{*}$ & $1.30 \pm 0.58$ & $1.14 \pm 0.46$ \\
& Finger & $2.31 \pm 1.45$ & $1.14 \pm 0.46^{*}$ & $1.78 \pm 0.88$ & $1.46 \pm 0.55$ \\
Vertical direction & Shoulder & $0.34 \pm 0.17$ & $0.38 \pm 0.34$ & $0.46 \pm 0.27$ & $0.32 \pm 0.17$ \\
(z-axis) & Wrist & $1.16 \pm 0.47$ & $0.85 \pm 0.19$ & $1.25 \pm 0.86$ & $0.86 \pm 0.23$ \\
& Finger & $1.91 \pm 0.90$ & $1.27 \pm 0.39$ & $1.60 \pm 1.27$ & $1.17 \pm 0.27$ \\
\hline
\end{tabular}

Data are expressed as mean $\pm \mathrm{SD}$.

$* \mathrm{p}<0.05$, within group, ${ }^{\dagger} \mathrm{p}<0.05$, between groups

Table 3. Effects of craniocervical flexion exercise on the velocity of trajectories

\begin{tabular}{lccccc}
\hline & & \multicolumn{2}{c}{ Exercise group $(\mathrm{cm} / \mathrm{s})$} & \multicolumn{2}{c}{ Control group $(\mathrm{m} / \mathrm{s})$} \\
\hline Lateral direction & Phe & Post & Pre & Post \\
(y-axis) & Wrist & $0.41 \pm 0.12$ & $0.27 \pm 0.07^{* \dagger}$ & $0.50 \pm 0.20$ & $0.42 \pm 0.11$ \\
& Finger & $0.95 \pm 0.30$ & $0.75 \pm 0.15^{*}$ & $0.81 \pm 0.28$ & $0.81 \pm 0.15$ \\
Vertical direction & Shoulder & $0.21 \pm 0.11$ & $0.15 \pm 0.02$ & $0.26 \pm 0.18$ & $0.18 \pm 0.05$ \\
(z-axis) & Wrist & $0.68 \pm 0.17$ & $0.67 \pm 0.12$ & $0.67 \pm 0.18$ & $0.63 \pm 0.12$ \\
& Finger & $0.87 \pm 0.29$ & $0.77 \pm 0.18$ & $0.76 \pm 0.32$ & $0.80 \pm 0.13$ \\
\hline
\end{tabular}

Data are expressed as mean \pm SD.

${ }^{*} \mathrm{p}<0.05$, within group, ${ }^{\dagger} \mathrm{p}<0.05$, between groups

Several studies report craniocervical flexion exercise is a useful method for retraining the deep cervical flexor muscles, increasing stability, and enhancing head and neck proprioception ${ }^{2,3)}$. Sanchez et al. $\left.{ }^{6}\right)$ report that the alteration of proprioception might affect postural stability. The finding that craniocervical flexion exercise reduced tremor suggests this exercise might contribute to postural stability.

In conclusion, craniocervical flexion exercise reduces the range and velocity of tremor, thereby increasing postural stability. This result has important implications in physical therapy protocols for patients with poor postural control. However, further complementary studies with diverse methods are required to confirm the present results.

\section{ACKNOWLEDGEMENT}

This research was supported by the Basic Science Research Program through the National Research Foundation of Korea (NRF) funded by the Ministry of Science, ICT \& Future Planning (No. 2013R1A1A3007734).

\section{REFERENCES}

1) Shumway-Cook A, Woollacott MH: Motor control: translating research into clinical practice, Lippincott Williams \& Wilkins, 2007.

2) Armstrong BS, McNair PJ, Williams M: Head and neck position sense in whiplash patients and healthy individuals and the effect of the craniocervical flexion action. Clin Biomech (Bristol, Avon), 2005, 20: 675-684. [Medline] [CrossRef]

3) Falla DL, Jull GA, Hodges PW: Patients with neck pain demonstrate reduced electromyographic activity of the deep cervical flexor muscles during performance of the craniocervical flexion test. Spine, 2004, 29: 2108-2114. [Medline] [CrossRef]

4) Treleaven J: Sensorimotor disturbances in neck disorders affecting postural stability, head and eye movement control. Man Ther, 2008, 13: 2-11. [Medline] [CrossRef]

5) Harman K, Hubley-Kozey CL, Butler H: Effectiveness of an exercise program to improve forward head posture in normal adults: a randomized, controlled 10-week trial. J Manual Manip Ther, 2005, 13: 163-176. [CrossRef]

6) Sanchez-Ramirez DC, van der Leeden M, Knol DL, et al.: Association of postural control with muscle strength, proprioception, self-reported knee instability and activity limitations in patients with knee osteoarthritis. J Rehabil Med, 2013, 45: 192-197. [Medline] [CrossRef]

7) Lluch E, Schomacher J, Gizzi L, et al.: Immediate effects of active craniocervical flexion exercise versus passive mobilisation of the upper cervical spine on pain and performance on the cranio-cervical flexion test. Man Ther, 2014, 19: 25-31. [Medline] [CrossRef]

8) Jull G, Barrett C, Magee R, et al.: Further clinical clarification of the muscle dysfunction in cervical headache. Cephalalgia, 1999, 19: 179-185. [Medline] [CrossRef]

9) Kisner C, Colby LA: Therapeutic exercise: foundations and techniques, FA Davis, 2012.

10) Pellegrini B, Faes L, Nollo G, et al.: Quantifying the contribution of arm postural tremor to the outcome of goal-directed pointing task by displacement measures. J Neurosci Methods, 2004, 139: 185-193. [Medline] [CrossRef]

11) Kerr G, Morrison S, Silburn P: Coupling between limb tremor and postural sway in Parkinson's disease. Mov Disord, 2008, 23: 386-394. [Medline] [CrossRef]

12) Iqbal ZA, Rajan R, Khan SA, et al.: Effect of deep cervical flexor muscles training using pressure biofeedback on pain and disability of school teachers with neck pain. J Phys Ther Sci, 2013, 25: 657-661. [Medline] [CrossRef]

13) Silva AG, Johnson MI: Does forward head posture affect postural contro in human healthy volunteers? Gait Posture, 2013, 38: 352-353. [Medline] [CrossRef]

14) Lee MY, Lee HY, Yong MS: Characteristics of cervical position sense in subjects with forward head posture. J Phys Ther Sci, 2014, 26: 1741-1743. [Medline] [CrossRef] 